\title{
Adaptive silver films for surface-enhanced Raman spectroscopy of biomolecules
}

\author{
Vladimir P. Drachev ${ }^{1}$, Mark D. Thoreson ${ }^{1 *}$, Vishal Nashine ${ }^{3}$, Eldar N. Khaliullin ${ }^{1}$, \\ Dor Ben-Amotz ${ }^{2}$, V. Jo Davisson ${ }^{3}$, Vladimir M. Shalaev ${ }^{1}$ \\ ${ }^{1}$ School of Electrical and Computer Engineering, ${ }^{2}$ Department of Chemistry, and \\ ${ }^{3}$ Department of Medicinal Chemistry and Molecular Pharmacology \\ Purdue University, West Lafayette, IN 47907 \\ ${ }^{*}$ Corresponding author: 465 Northwestern Ave, West Lafayette, IN 47907 , \\ tel: +1 765 494-0537, fax: +1 765 494-6951, mthoreso@purdue.edu
}

\begin{abstract}
The interaction of biological molecules with a typical substrate for surface-enhanced Raman scattering (SERS) often leads to their structural and functional changes. In this work we describe SERS substrates, called adaptive silver films (ASFs), in which the biomaterial and the substrate act in concert to produce excellent Raman enhancement through local restructuring of the metal surface while at the same time preserving the properties (such as conformational state and binding activity) of the analyte. These adaptive substrates show great promise for SERS spectroscopy of many different types of biomolecules, and we provide several current examples of their use.
\end{abstract}

\section{Introduction}

One of main advantages of Raman scattering as a detection method for molecule sensing is well known. Raman spectra enable fingerprinting of molecules which is of particular interest for bio-applications. Surface enhanced Raman scattering (SERS) provides greater detection sensitivity than conventional Raman spectroscopy [1-3], and it is quickly gaining traction in the study of biological molecules adsorbed on a metal surface [4-12]. SERS spectroscopy allows for the detection and analysis of minute quantities of analytes because it is possible to obtain high-quality SERS spectra at submonolayer molecular coverage as a result of the large scattering enhancements. SERS has also been shown to be sensitive to molecular orientation and to the distance from the metal surface [13]. Thus, SERS is well-suited for biomolecule studies in which specificity and sensitivity to the conformational state and orientation of the molecule are very important.

The SERS enhancement mechanism originates in part from the large local electromagnetic fields caused by resonant surface plasmons that can be optically excited at certain wavelengths for metal particles of different shapes or closely spaced groups of particles [14-21]. For aggregates of interacting particles, which are often structured as fractals, plasmon resonances can be excited in a very broad spectral range [22]. In addition to electromagnetic field enhancement, metal nanostructures and molecules can form charge-transfer complexes that provide further enhancement for SERS [23-29]. The resulting overall enhancement depends critically on the particle or aggregate nanostructure morphology [22,30-36], and it can be as high as $10^{5}$ to $10^{8}$ for 
the area-averaged macroscopic signal and $10^{10}$ to $10^{15}$ within the local resonant nanostructures.

A variety of structures have been found to be appropriate for SERS, including roughened metal electrodes [1-3], aggregated films [15], metal islands of different morphology [14,15,17-20], and semicontinuous films near the percolation threshold [3739]. Among SERS-active substrates, vacuum-evaporated nanostructured metal films are well suited for SERS mechanism studies and have a high potential for applications [14-20,24,39-43]. The effect on the metal film due to deposition rate, mass thickness, and thermal annealing were studied in detail previously [18,40-43]. We found recently that silver films fabricated at a certain range of evaporation parameters allow fine rearrangement of their local structure under protein deposition [44]. Such a substrate, which is referred as adaptive, provides large SERS enhancement and appears to be biocompatible. In this work we discuss specific properties of adaptive substrates and provide several examples of their uses for protein sensing. We also describe further development of this type of substrate to improve SERS signal by addition of a sub-layer of the bulk metal.

\section{Adaptive Silver Films}

Although most SERS substrates have a predefined and static metal-dielectric nanostructure, this is not in general the best case for obtaining an optimal Raman signal from any particular analyte. Experiments reveal that a variation of evaporated metal films produces substrates with new adsorption and restructuring features that are important for SERS detection and analysis of biomolecules [44]. In particular, the restructuring involves a competition of two processes. A protein solution (e.g. in Tris buffered saline) slightly dissolves Ag particle surfaces to make them movable, and stabilization with proteins. Evaporated metal films that allow restructuring are herein called adaptive to distinguish them from static-structure films. As we have predominantly used silver as the metal in our films in order to take advantage of theoretically higher enhancements from silver, we will refer to these special substrates as adaptive silver films (ASFs). The ASFs are typically formed on a dielectric substrate under vacuum evaporation with an electron beam (see details in [45]). The dielectric (glass) slides were covered first by a sub-layer of $10 \mathrm{~nm}$ of $\mathrm{SiO}_{2}$ followed by an 8-13 nm $\mathrm{Ag}$ layer deposited at a rate of $0.05 \mathrm{~nm} / \mathrm{s}$. During the silver deposition process, small isolated metal granules are formed first on the dielectric substrate. As the silver coverage increases, the granules coalesce, resulting in various sizes of silver particles and their aggregates.

Along with the advantages provided by being SERS-active, these adaptive films address three additional issues. First, direct adsorption of biomolecules on a metal surface often leads to their significant structural (denaturation) and/or functional changes $[5,8,46,47]$. Hence, not all substrates are biocompatible while it is of great interest to study biomolecules in their native forms with functionality preserved. Second, any particular metal nanostructure with a fixed morphology cannot perfectly match different analytes and provide the optimal SERS in all cases due to the differences in analyte sizes. Lastly, biomolecular research often involves several washing procedures to remove non-binding or excess agents. This washing can also remove metal nanoparticles with medium to low adhesion to the substrate. Adaptive 
silver films allow protein-mediated restructuring of the nanoparticles, which makes it possible to address all three issues simultaneously. Specifically, biomolecules adsorb on the metal surface without significant structural changes (soft-adsorption), the silver film stabilizes which makes the analyte/metal combinations resistant to washing, and the SERS signal improves for given set of particles.

The examination of these ASF substrates involves characterization of the substrates by many methods, including UV-Vis spectrophotometry, field emission scanning electron microscopy, adhesion testing, atomic force microscopy, and SERS enhancement. We will discuss several of these methods individually, and we will also include some discussion on the visible characteristics before and after analyte solution deposition on ASF substrates.

\subsection{Film stabilization}

A representative view of an ASF substrate before and after proteins deposition is shown in Fig. 1. Note that after washing with Tris-buffered saline (TBS) Tween-20 solution, the silver coating has been removed everywhere except the areas under the analyte spots, as clearly seen in Fig. 1. From this we can see that the biomolecules stabilize the silver film, allowing the silver film to remain in place even through washing procedures. This indicates that the biomolecules themselves play a key role in forming a stable complex with the silver particles. By varying the biomolecule and buffer concentrations, we observe that both factors are important in the formation of uniform, stabilized analyte spots]. A lower biomolecule concentration (by roughly a factor of 10-20) in our deposition solution results in an almost transparent spot and hence no metal particles. The solvent may dissolve the metal particles, leaving merely silver salt on the substrate. This process may affect the interface between $\mathrm{Ag}$ particles and silica and decrease adhesion or even remove particles in solution. Deposition of the biomolecule solution without buffer reveals no visible changes of the film surface. We also verified in a test experiment that film restructuring and stabilization can also be done with a synthetic polymer like polyvinylpirrolidone (PVP) in TBS solution.

\subsection{UV-VIS Spectral Analysis}

Excitation of the collective electron oscillations (plasmons) in a metal nanostructure results in strong light and particles interaction and eventually in increased absorption relative to thick metal film. Near-ultraviolet and visible spectra are measured using a spectrophotometer to characterize nanostructured films. In particular, the reflectance and absorbance spectra are compared to provide information on the film structure. The light impinging on a semicontinuous metal film will undergo transmission, reflection, or absorption, with a small percentage $(<5 \%)$ experiencing scattering. By measuring the intensity of light in each of these processes at different frequencies, one can judge whether film the structure supports the resonant plasmon modes. At the frequencies where resonances occur, absorption increases. Typical UV-VIS absorbance and reflectance spectra of an ASF substrate are similar in shape and have a maximum at around $500 \mathrm{~nm}$ with a broad wing into the longer wavelengths (see Fig. 2). Reflection is typically comparable or slightly larger than absorption (by a factor of roughly 1-1.4 when both spectra are expressed in percent). Both the visible color of the film and the extinction spectrum change after analyte deposition, as is shown in Fig. 2. The 
spectrum inside the analyte spot typically shows a blue-shifted maximum, reduced slope of the long wavelength wing, and reduced extinction integrated over the $300 \mathrm{~nm}$ to $1100 \mathrm{~nm}$ spectral range.

\subsection{FE SEM Image Analysis}

One of the best ways to characterize the ASF substrates is in actually looking at the nanostructure of the metal film. A scanning electron microscope (SEM) is potentially useful here, but they often have problems with charging for non-conductive samples such as semicontinuous films on dielectric substrates (like ASFs). A better method for ASFs is the use of a field emission SEM (FE SEM). Using such a device, it is possible to obtain excellent views of the ASF nanostructure and to determine changes in the nanostructure as a result of the analyte deposition and restructuring.

Representative nanostructure images before and after analyte deposition are shown in Fig. 3 for an $11 \mathrm{~nm}$ ASF. In this particular case M2 monoclonal antibody of about $1 \mu \mathrm{M}$ in TBS solution was deposited. The images show metal nanoparticles and their aggregates in white, while dielectric material is dark gray or black. The large-sized particles have complicated shapes an internal structure. The figure clearly shows that the film experiences nanoscale restructuring inside the biomolecule spot, where groups of closely spaced metal nanoparticles are formed. This is in contrast to the film outside the biomolecule spot, where rather disintegrated particles are typical. A metal filling factor can also be calculated from FE SEM images. Defined as the ratio of the area covered by metal to the total area, the filling factor is lower inside the biomolecule spot than outside (see Fig 3). Decreases in the integrated extinction and in the metal filling factor both suggest a decrease of the silver mass thickness caused by biomolecule deposition. This indicates that some of the metal nanoparticles have been dissolved in solution. It is interesting that extinction changes mostly after protein deposition and does not change much after subsequent washing.

\subsection{Atomic Force Microscopy}

While FE SEM data gives us information on the lateral structure of the film, AFM analysis yields insight into the vertical structure of the ASF. A typical AFM height profile for an ASF is shown in Fig. 4. AFM analysis indicates that the particle height is typically less than their lateral plane size. The maximal height exceeds mass thickness which is expected for nanostructured film. An example of an $8 \mathrm{~nm}$ film shows maximal height of about $30 \mathrm{~nm}$ with RMS deviations from the mean in the range of 5-7 nm. At the same time the ASF is uniform on the scale of micrometers and higher so that this film is very homogeneous within a laser spot of about 80-100 $\mu \mathrm{m}$.

\subsection{Adhesion Testing}

The adhesion of the metal film to the glass substrate is important to the function of our ASF films. If adhesion is too high, no discernable restructuring will occur. At the same time, very low adhesion yields poor film quality and stability before analyte deposition. A quantitative adhesion analysis of these semicontinuous films is difficult to implement, but qualitative estimates of adhesion were performed with the common tape test [48]. In this test, the tape was attached to the film and then pulled off. Absorption and reflection measurements before and after the tape test indicate the relative level of 
adhesion of the film to the glass substrate. A comparison of silver substrates with and without an $\mathrm{SiO}_{2}$ sub-layer show that adhesion of silver on glass is poor, while the $\mathrm{Ag} / \mathrm{SiO}_{2} /$ glass substrate shows good adhesion in the tape test. The changes in absorption/reflection spectra were less than $5 \%$ for the $\mathrm{Ag} / \mathrm{SiO}_{2} / g$ lass substrate [44]. In the case of high adhesion (such as including an adhesion-promoting titanium sub-layer), the deposition of the biomolecule solution does not lead to spot color changes nor structural modifications, and typically little or no SERS is observed.

\subsection{XPS Analysis}

X-ray photoelectron spectroscopy (XPS) is used to analyze the elemental species on a sample surface based on the photoelectron effect. This analysis technique is capable of probing roughly $10 \mathrm{~nm}$ of a sample surface. In the case of ASF samples, this depth corresponds approximately to the metal film thickness, and we can estimate that the analysis results include predominantly data from the outer metal layer along with any biomolecules deposited onto the film surface. By measuring kinetic energy of the photoelectrons at a given photon energy $(1486.6 \mathrm{eV})$, one can detect the binding energy spectrum. The binding energies of the peaks are characteristic for each element. The peak areas can be used to determine the relative composition of the sample surface. The shape of each peak and the binding energy can be slightly altered by the chemical state of the emitting atom. Hence XPS can provide chemical bonding information as well. In our case the high energy resolution spectra provide information on the oxidation state of silver and the relative composition of different carbon bonds, including hydrocarbons, $\mathrm{C}-\mathrm{C}, \mathrm{C}-\mathrm{O}(\mathrm{C}-\mathrm{N})$ bonds, and $\mathrm{O}-\mathrm{C}=\mathrm{O}$.

Our XPS analysis was performed through Rocky Mountain Labs, Inc., for several ASF samples. The results indicate the relative amounts of elemental species in our metal films and in the analytes deposited on those films. In our vacuum-evaporated films studied with XPS, roughly $25-30 \%$ of the composition is silver, while carbon makes up $30-35 \%$. Oxygen (25-28\%), silicon (12-14\%), and sodium $(<2 \%)$ are also present on the surface. The silicon, oxygen in part, and sodium are most likely attributable to the glass or silicon dioxide sublayers in the ASF substrate structure. Representative XPS high energy resolution spectrum of the $\mathrm{Ag} 3 \mathrm{~d}$ region is shown in Fig.5. An important result is that the silver on the substrate is still in its metal state without oxidation after 23 weeks from fabrication, while the metal is in an oxidized state about eight weeks from fabrication. The presence of metal-state $\mathrm{Ag}$ indicates that the substrates are reasonably stable over time, which is very helpful for production and storage of the substrates. Deposition of a protein solution makes silver surface partially oxidize.

XPS analysis also indicates that there is carbon contamination on the substrates. This is typical of vacuum-evaporated films, and very difficult to avoid. We also note that there is no contamination from other evaporation sources (such as gold or titanium used in the vacuum evaporator previously) in our samples, which indicates that the evaporator system is relatively clean and the source purity is very high.

\subsection{SERS Enhancement}

The biomolecule-mediated restructuring illustrated by FE SEM images in Fig. 3 results in aggregates of closely spaced particles covered with biomolecules. This is in contrast to the rather disintegrated particles of the initial film before analyte deposition. 
Depending on the mass thickness of the initial film, small or large fractal-like aggregates can be formed. The analyte SERS signal, normalized per metal mass coverage, is comparable for both the small and large aggregates that we examined. SERS enhancement is high enough to detect a monolayer of analyte [44]. Note that an aggregated structure provides conditions for both electromagnetic and chemical SERS enhancements. Even small aggregates provide strong electromagnetic enhancement in the visible and near infrared, as has been shown for polarization nonlinearities [49] and SERS [31]. Large aggregates typically have a fractal morphology, which is known to provide a particularly strong SERS signal $[22,39]$. In addition, the first molecular layer may also produce conditions to make an optical tunnel current possible, either through the molecule at the point of nearest approach between two particles [39], or through a system operating as a molecular tunnel junction between particles across a vibrating molecular bridge [50]. Thus, adaptive feature of our films produces cavity sites enclosed by two or more particles, which are optimal for enhanced Raman. It is even more important that the cavity sites are naturally filled with proteins as a result of restructuring.

The measured macroscopic enhancement factor for insulin on our ASF substrate relative to normal Raman of insulin on quartz is about $3 \times 10^{6}$, which is among the largest observed for random metal-dielectric films [44].

It is important to note that, in general, non-adaptive films (without restructuring) are created by optimizing either evaporation parameters (sub-layer, deposition rate, increased mass thickness) or biomolecule solution contents. This means that nonadaptive substrates should be tailored to specific biomolecule solutions, or vice-versa. Clearly such changes can affect metal structures or biomolecule adsorption, which makes it impossible to directly compare SERS results on adaptive films to those from non-adaptive films. Regardless, from analysis of FE SEM images and SERS enhancement results, the observation is that there is no detectable SERS without restructuring.

\section{Biomolecule detection with ASFs}

In order to demonstrate the use of ASF substrates for SERS of biomolecules, we provide two examples. We first discuss ASF experiments involving the detection of insulin analogs. Next we cover the detection of antibody-antigen binding events using ASF substrates.

Insulin is a protein consisting of 51 amino acids split into two chains, as shown in Fig. 6 , and is a glucose regulation agent in the bloodstream. In general, protein sensing using Raman spectroscopy provides important structural information on conformational changes. Changes between native and denatured insulin in the solid form as well as spectral features of proinsulin and insulin fibrils were studied previously [51-53]. Signatures of allosteric conformation changes in hexameric insulin have been assessed using Raman difference spectroscopy [54].

In our insulin analog experiments, we have demonstrated [44] that ASFs can be used for the observation of differences in SERS spectra of recombinant human insulin and insulin lispro at very low protein surface densities. As mentioned previously, the ASFs modify their local nanostructure under protein deposition so that the conformational state of insulins is preserved and SERS is improved. 
The experiments examined differences in Raman spectra of two insulin isomers, human insulin and its analog insulin lispro. These two insulins differ only in the interchange of two neighboring amino acids; specifically, the propyl-lysyl sequence at the $\mathrm{C}$-terminus of the B-chain in insulin lispro is inverted as compared to human insulin (see Fig. 6, near the C-terminus). This propyl-lysyl switch leads to conformational changes at the $\mathrm{C}$ - and $\mathrm{N}$-termini and has an important clinical effect for diabetes treatment. The difference in SERS spectra for the two insulins (see Fig. 8) was detected at a sub-monolayer density of $80 \mathrm{fmol} / \mathrm{mm}^{2}$, with only 25 amol in the probed area. Our SERS spectra indicate that insulin is adsorbed on the metal surface through $\mathrm{N}$ termini. This suggests a possible scenario for insulin and silver particle interaction on the surface and helps us understand the restructuring mechanism in this case. Because of specific $\mathrm{Cl}^{-1}$ adsorption, silver particles acquire excess negative charges and attract the positively charged $\mathrm{N}$-terminus. Insulin hexamers have six $\mathrm{N}$-termini of $\mathrm{B}$ chains exposed to the hexamer surface, three at the top and three at the bottom [44]. Thus, insulin hexamers can serve as coupling agents and induce the surface restructuring within a protein spot.

Since human insulin and its analog have the same set of side chains and differ only in conformational states, the observed difference indicates the preservation of the conformational state with the use of adaptive silver substrates. In this study we used Raman difference spectroscopy, which is a general method of probing protein structure for comparison between closely related proteins [55], which we extended to SERS in order to study the spectral features of insulin conformation. In our experiments, all insulin vibration modes are enhanced by approximately the same factor. This makes the SERS spectra similar to the conventional Raman spectra in liquid and solid forms and simplifies the analysis.

We have also studied ASF substrates for use in antibody/antigen binding research. The detection of protein binding by optical means is of critical importance to current protein analysis, and promises to lead to a number of exciting future applications in biomedical diagnostics, research and discovery. Our results show that SERS substrates based on ASFs allow direct, label-free SERS detection of Ab-Ag binding at a monolayer level. In experiments we used the following protocol. Antigens (C-terminal FLAG-BAP (bacterial alkaline phosphatase) were immobilized on ASF substrates to form a monolayer array, followed by incubation with an antibody (anti-FLAG M2 monoclonal antibody) solution at $1 \mathrm{nM}$ concentration. Each time, after immobilization of $\mathrm{Ag}$ and incubation with $\mathrm{Ab}$, the film was washed for about $20 \mathrm{~min}$ in a solution of Trisbuffered saline (TBS) containing $0.5 \%$ Tween-20 and then rinsed 5 times with deionized water. After washing, SERS spectra were collected to observe a spectral change following the incubation of $\mathrm{Ab}$ with Ag. Antigen-antibody binding event results in distinct SERS spectral changes as shown in Fig. 9. It is important that the ASF substrate allow independent in situ binding activity validation using traditional chemiluminescence and fluorescence methods. We performed such validation and confirmed that antigens and antibodies retain their binding properties on our SERS-active substrate. As with insulin, we find that the deposited biomolecules (antibodies or antigens in this case) restructure and stabilize the ASFs so that the protein binding activities are preserved and, in parallel, SERS is improved. 
Label-free detection using the ASF method produces unique advantages relative to prior optical binding detection methods (typically based on different types of labels) such as scintillation counting [56], electrochemical [57], enzymatic [58], fluorescence [59-60], and chemiluminescence methods [61]. An additional feature of ASFs is an ability to employ various detection methods on the same substrate, such as label-free SERS, chemiluminescence, fluorescence, and further analysis of proteins retrieved from the substrate spot.

\section{Multi-layer ASF structures}

A metal nanostructured film positioned near a mirror-like metal surface with a sandwiched dielectric layer can show dramatic change in the film's optical properties [62-64]. The localized plasmon resonances supported by the constituents of metal island films experience a frequency shift when placed in close proximity to a conducting surface [62]. The strong dependence of the spectral position of the reflectivity minimum on the spacer thickness is caused by enhanced absorption [63]. The enhancements in absorption and elastic scattering [64] are likely associated with the existence of strongly enhanced local fields. These changes are attributed to an enhancement of the dipoledipole interaction between the different particles mediated by propagating modes that are supported by a layered sample [64], interaction between dipole and its own image [62], and interference effects between waves reflected from different interfaces in the sample [63].

We employ such a sandwich structure to further increase SERS signal from ASF based biosensors. The sandwich structure (Fig. $9 \mathrm{~b})$ contains a bulk silver layer $(80 \mathrm{~nm})$ deposited on glass, then a layer of $\mathrm{SiO}_{2}(10 \mathrm{~nm})$, and finally a $12 \mathrm{~nm}$ nano-structured silver film. So, relative to the usual ASF (Fig. 9a) it has an additional sublayer of bulk metal. The bulk silver layer provides an additional enhancement of the local fields caused by interaction between particles and their images in the bulk layer and far-field interactions between particles. We performed test experiments with three analytes: human insulin, anti-human interleukin 10, and anti-human interleukin 10 incubated with $1 \mathrm{nM}$ R6G. Fig. 10 illustrates the relative increment of the SERS intensity for R6G inside an antibody (anti-human interleukin 10) spot. Table 1 shows specific intensities (in counts per second per $\mathrm{mW}$ ) of a characteristic peak of $A b$ and R6G from the sandwich ASF sample and the usual ASF structure. Note that in our experiments with insulin we observed $28-38 \mathrm{c} / \mathrm{smW}$ from the sandwich structure (vs $1.5-4 \mathrm{c} / \mathrm{smW}$ for the particular substrate used for comparison and $7 \mathrm{c} / \mathrm{smW}$ for maximal signal over all samples). From the data in Table 1 we conclude that the multi-layer sandwich structure provides a signal increment of roughly $4-5$ at least.

\section{Summary}

In summary, the nanostructured adaptive silver films (ASFs) demonstrate advantages over static-structure SERS substrates. The characterization of these films leads to the conclusion that ASFs experience fine restructuring under biomolecule deposition such that conformation and functionality are preserved. In addition, the biomoleculemediated restructuring produces excellent conditions for SERS enhancement. The interaction of the silver film with the biomolecule solution acts to stabilize the system, making it resistant to wash processes. 
Examples show that ASFs preserve secondary structure of insulin and have a high SERS sensitivity in detection of conformational distinctions of nearly identical insulin isomers. In addition, we find that SERS substrates based on ASFs can be used for direct, label-free detection of protein binding at a monolayer level. Finally, ASF substrates allow independent validation of $\mathrm{Ab} / \mathrm{Ag}$ by conventional chemiluminescence and fluorescence methods and show great promise for detection and analysis of biomolecules, even at very low surface densities.

Experiments with the a sandwich structure including a bulk metal layer and the adaptive silver film reveal a promising way to further improve sensitivity of SERS-based biosensors.

This research was sponsored in a part by a grant from Inproteo.

\section{References}

[1] Fleischmann M, Hendra PJ, McQuillan AJ. Chem. Phys. Lett. 1974; 26: 163.

[2] Jeanmaire DJ, Van Duyne RP. J. Electroanal. Chem. 1977; 84: 1.

[3] Albrecht MG, Creighton JA. J. Am. Chem. Soc. 1977; 99: 5215.

[4] Vo-Dinh T. Trends Anal. Chem. 1998; 17: 557.

[5] Bauer G, Stich N, Schalkhammer TGM. Methods and Tools in Biosciences and Medicine Analytical Biotechnology, Schalkhammer TGM, Ed.; Birkhauser Verlag Basel, Switzerland, 2002.

[6] Sibbald MS, Chumanov G, Cotton TM. J. Electroanal. Chem. 1997; 438: 179.

[7] Vo-Dinh T, Stokes DL, Griffin GD, Volkan M, Kim, UJ, Simon MI. J. Raman Spec. 1999; 30: 785.

[8] Brown KR, Fox AP, Natan MJ. J. Am. Chem. Soc. 1996; 118: 1154.

[9] Shafer-Peltier KE, Haynes CL, Glucksberg MR, Van Duyne RP. J. Am. Chem. Soc. 2003; 125: 588.

[10] Cao YWC, Jin R, Mirkin CA. Science 2002; 297: 1536.

[11] Cao YC, Jin R, Nam JM, Thaxton CS, Mirkin CA. J. Am. Chem. Soc. 2003; 125: 14676.

[12] Grubisha DS, Lipert RJ, Park HY, Driskell J, Porter, MD. Anal. Chem. 2003; 75: 5936-5943.

[13] Moskovits M. Rev. Mod. Phys. 1985; 57: 783.

[14] Moskovits M. J. Chem. Phys. 1978; 69: 4159.

[15] Chen CY, Burstein E, Lundquist S. Solid State Commun. 1979; 32: 63.

[16] McCall SL, Platzman PM, Wolff PA. Physics Lett. 1980; 77A: 381.

[17] Chen CY, Burstein E. Phys. Rev. Lett. 1980; 45: 1287.

[18] Bergman JG, Chemla DS, Liao PF, Glass AM, Pinczuk A, Hart RM, Olson DH. Optics Lett. 1981; 6: 33.

[19] Weitz DA, Garoff S, Gramila TJ. Optics Lett. 1982; 7: 168.

[20] Ritchie G, Chen CY. Surface Enhanced Raman Scattering, Chang PK, Furtak TE, Eds., Plenum: New York, 1982, p361.

[21] Schatz GC. Fundamentals and Applications of Surface Raman Spectroscopy, Garrell RL, Pemberton JE, Cotton TM, Eds., VCH Publishers: Deerfield Beach, FL, 1993. 
[22] Stockman MI, Shalaev VM, Moskovits M, Botet R, George TF. Phys. Rev. B 1992; 46: 2821.

[23] Otto A. Surf. Science 1978; 75: 1392.

[24] Pockrand I, Otto A. Solid State Commun. 1980; 35: 861.

[25] Persson BNJ. Chem. Phys. Lett. 1981; 82: 56.

[26] Adrian FJ. J. Chem. Phys. 1982; 77: 5302.

[27] Pandey PK, Schatz GC. J. Chem. Phys. 1984; 80: 2959.

[28] Lombardi JR, Birke RL, Lu T, Xu J. J. Chem. Phys. 1986; 84: 4174.

[29] Campion A, Kambhampti P. Chem. Soc. Rev. 1998; 27: 241.

[30] Nie S, Emory SR, Science 1997; 275: 1102. J. Phys. Chem. B 1998; 102: 493.

[31] Kneipp K, Wang Y, Kneipp H, Perelman LT, Itzkan I, Dasari RR, Feld M. Phys. Rev. Lett. 1997; 78: 1667.

[32] Schatz GC, Van Duyne RP. Handbook of Vibrational Spectroscopy, Chalmers JM, Griffiths RP, Eds., Wiley: New York, 2002, p 759.

[33] Musick MD, Keating CD, Keefe MH, Natan MJ. Chem. Mater. 1997; 9: 1499.

[34] Michaels AM, Nirmal M, Brus LE. J. Am. Chem. Soc. 1999; 121: 9932.

[35] Haynes CL, Van Duyne RP. J. Phys. Chem. 2003; 107: 7426.

[36] Prodan E, Radloff C, Halas NJ, Nordlander P. Science 2003; 302: 419.

[37] Gadenne P, Gagnot D, Masson M. Physica A 1997; 241: 161.

[38] Sarychev AK, Shalaev VM. Phys. Reports 2000; 335: 275.

[39] Shalaev VM. Nonlinear Optics of Random Media: Fractal Composites and MetalDielectric Films, STMP v.158, Springer: Heidelberg, 2000.

[40] Davies JP, Pachuta SJ, Cooks RG, Weaver M. J. Anal. Chem. 1986; 58: 1290.

[41] Schlegel VL, Cotton TM. Anal. Chem. 1991; 63: 241.

[42] Van Duyne RP, Hulteen JC, Treichel DA. J. Chem. Phys. 1993; 99: 2101.

[43] Vogel E, Kiefer W, Deckert V, Zeisel D. J. Raman Spec. 1998; 29: 693.

[44] Drachev VP, Khaliullin E, Thoreson MD, Davisson VJ, Shalaev VM. J. Physical Chemistry, in press. Drachev VP, Thoreson MD, Khaliullin EN, Sarychev AK, Zhang D, Ben-Amotz D, Shalaev VM, SPIE Proceedings, 2003; 5221: 76-81.

[45] ASF fabrication procedure: The microscope glass slides were cleaned through several steps, including multiple solvent rinses, a piranha $\left(\mathrm{H}_{2} \mathrm{O}_{2}: 3 \mathrm{H}_{2} \mathrm{SO}_{4}\right)$ acid bath, rinsing in $18 \mathrm{M} \Omega$ deionized water, and drying with pressurized gaseous nitrogen. Silver shot from Alfa Aesar (99.9999 \%, 1-3 mm) was used for fabrication of the ASFs on the glass substrates. The thin film deposition was performed in a high-vacuum electron-beam evaporator with an initial pressure inside the system of approximately $10^{-7}$ Torr. The film thickness and deposition rate were monitored with a quartz crystal oscillator. The glass slides were covered first by a sub-layer of $\mathrm{SiO}_{2}(10-12 \mathrm{~nm})$ followed by an Ag layer $(8-13 \mathrm{~nm})$ deposited at a rate of $0.05 \mathrm{~nm} / \mathrm{s}$. High-resolution FE SEM images were obtained through MAS Inc. (Raleigh, NC). AFM images were acquired with a Dimension 3100 (DI Veeco) using a $10 \mathrm{~nm} \mathrm{Si} \mathrm{tip} \mathrm{for} \mathrm{measuring} \mathrm{height} \mathrm{profiles} \mathrm{of} \mathrm{our}$ samples.

[46] Holt RE, Cotton TM. J. Am. Chem. Soc. 1989; 111: 2815.

[47] Yang M, Chang FL, Thompson M. Anal. Chem. 1993; 65: 3713.

[48] Eckertova L. Physics of Thin Films, Plenum Press: New York and London, 1977. 
[49] Drachev VP, Perminov SV, Rautian SG, Safonov VP. "Nonlinear optical effects and selective photomodification of silver colloidal aggregates", pp. 113-148, in Optical Properties of Nanostructured Random Media, Shalaev VM, Ed, Topics in Applied Physics, v. 82, Springer Verlag: Berlin, 2001.

[50] Michaels AM, Jiang JJ, Brus LE. J. Phys. Chem. B 2000; 104: 11965.

[51] Yu NT, Liu CS. J. Am. Chem. Soc. 1972; 94: 3250.

[52] Yu NT, Liu CS, O'Shea DC. J. Mol. Biol. 1972; 70: 117

[53] Yu NT, Jo BH, Chang RCC, Huber JD. Arch. Biochem. Biophys. 1974; 160: 614.

[54] Ferrari D, Diers JR, Bocian DF, Kaarsholm NC, Dunn MF. Biopolymers (Biospectroscopy) 2001; 62: 249-260.

[55] Callender R, Deng H, Gilmanshin R. J. Raman Spec. 1998; 29: 15.

[56] Gutcho S, Mansbach L. Clin. Chem. 1977; 23: 1609.

[57] Hayes FJ, Halsall HB, Heineman WR. Anal. Chem. 1994; 66: 1860.

[58] Butler JE. J. Immunoassay 2000; 21: 165.

[59] Vuori J, Rasi S, Takala T, Vaananen K. Clin. Chem. 1991; 37: 2087.

[60] Xu YY, Pettersson K, Blomberg K, Hemmila I, Mikola H, Lovgren T. Clin. Chem. 1992; 38: 2038.

[61] Brown CR, Higgins KW, Frazer K, Schoelz LK, Dyminski JW, Marinkovich VA, Miller SP, Burd JF. Clin. Chem. 1985; 31: 1500.

[62] Holland WR, Hall DG, Phys. Rev. Lett. 1984; 52: 1041.

[63] Leitner A, Zhao Z, Brunner H, Aussenegg FR, Wokaun A. Appl. Opt. 1993; 32: 102.

[64] Stuart HR, Hall DG. Phys. Rev. Lett. 1998; 80: 5663. 
Drachev et al, Figure 1

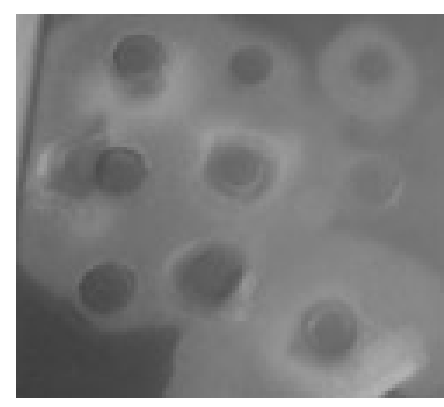

After deposition

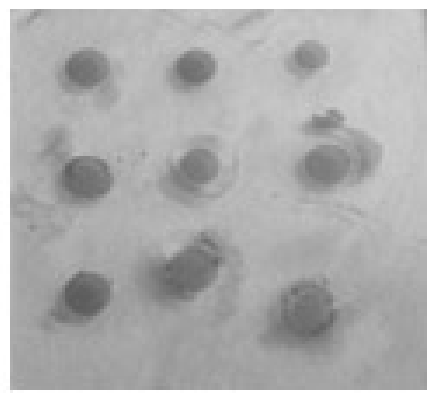

After washing

Fig. 1: ASF film with protein spots

before (top) and after (bottom) washing. 
Drachev et al, Figure 2

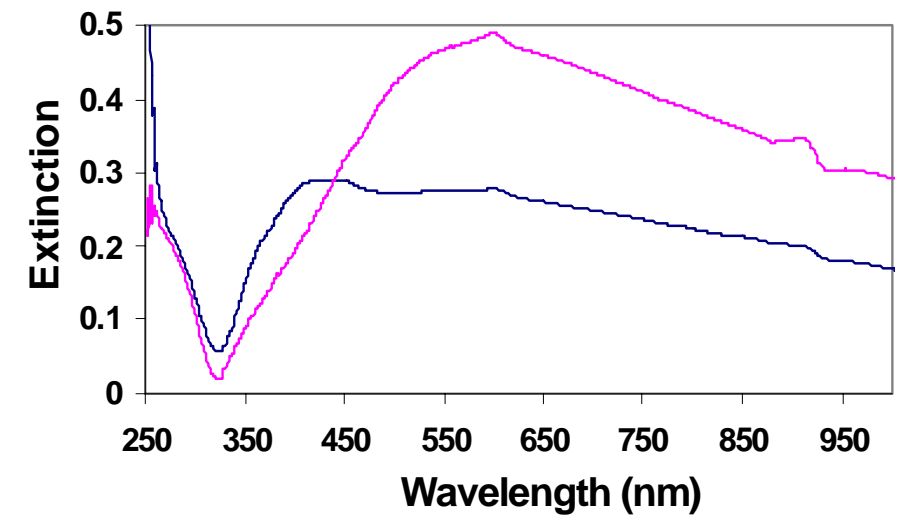

Fig. 2: Typical extinction spectra outside (red) and inside the protein spot (blue). 
Drachev et al, Figure 3

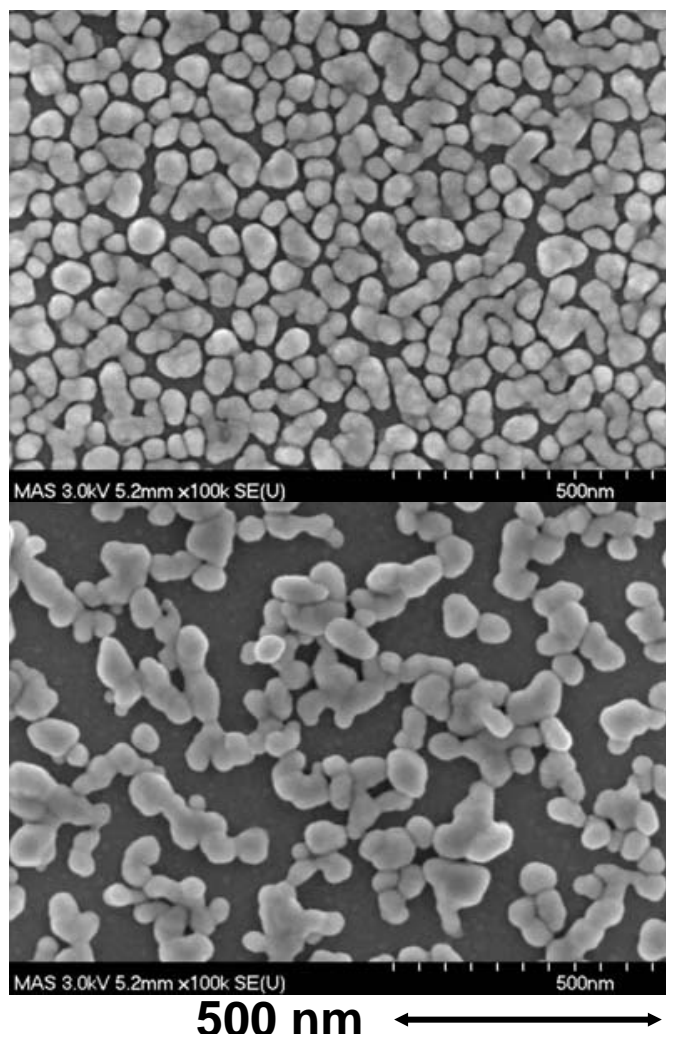

Fig. 3: FE SEM images of an ASF (11 nm mass thickness) inside spot before (top) and after protein deposition and washing (bottom) 
Drachev et al, Figure 4
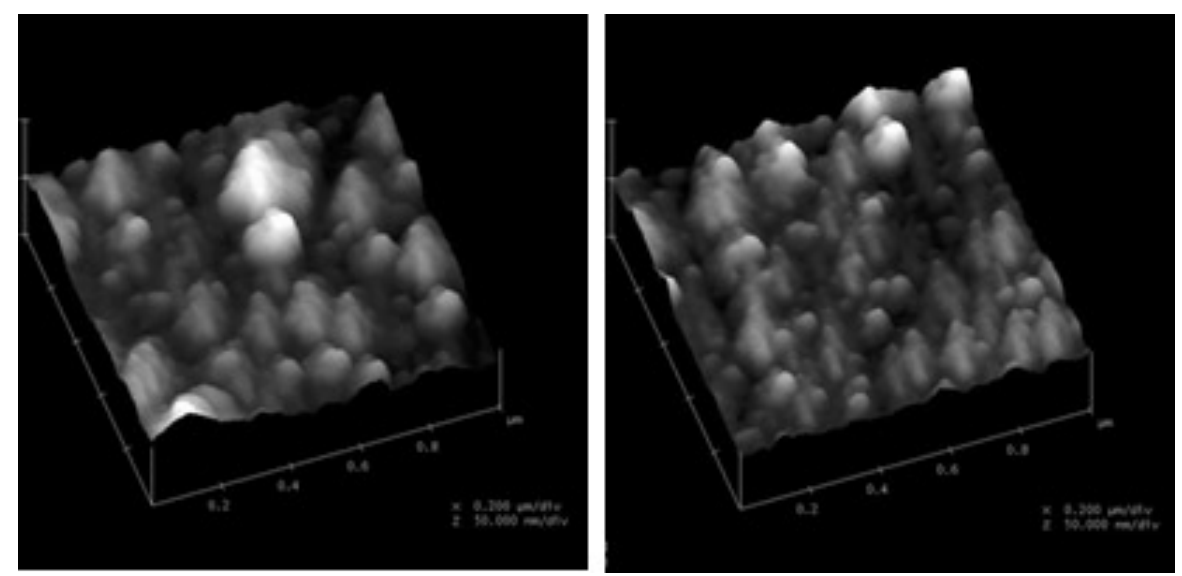

Fig. 4: AFM images of an 8-nm ASF before deposition (left) and after (right). 
Drachev et al, Figure 5

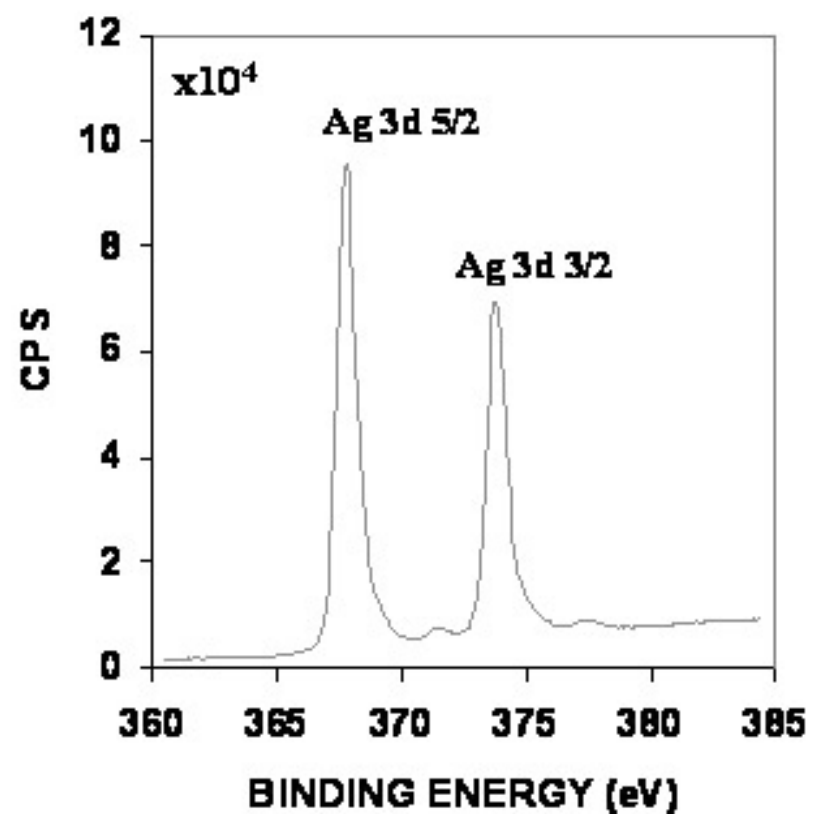

Fig.5: XPS High energy resolution spectrum of $\mathrm{Ag} 3 \mathrm{~d}$ region 
Drachev et al, Figure 6

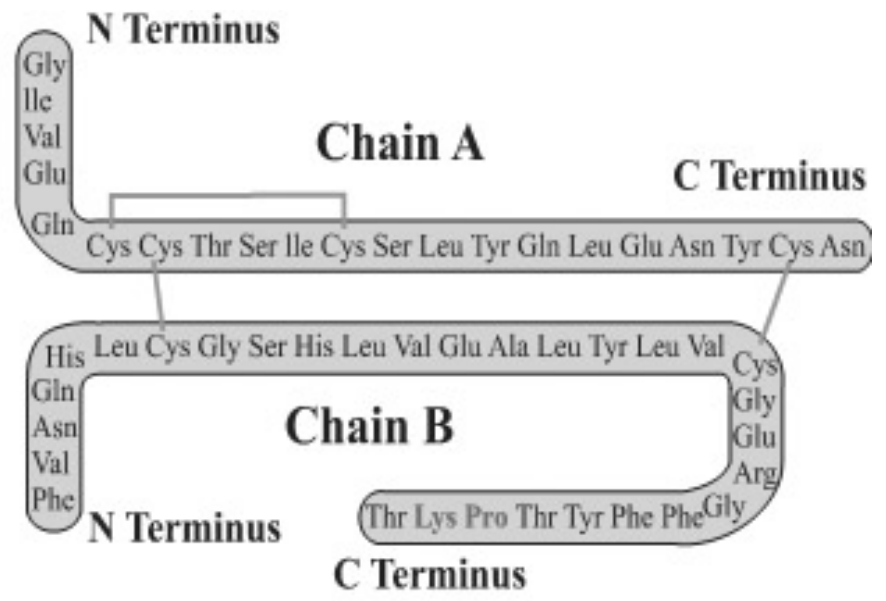

Fig. 6: Primary structure of human insulin, with two chains of 51 amino acids 
Drachev et al, Figure 7

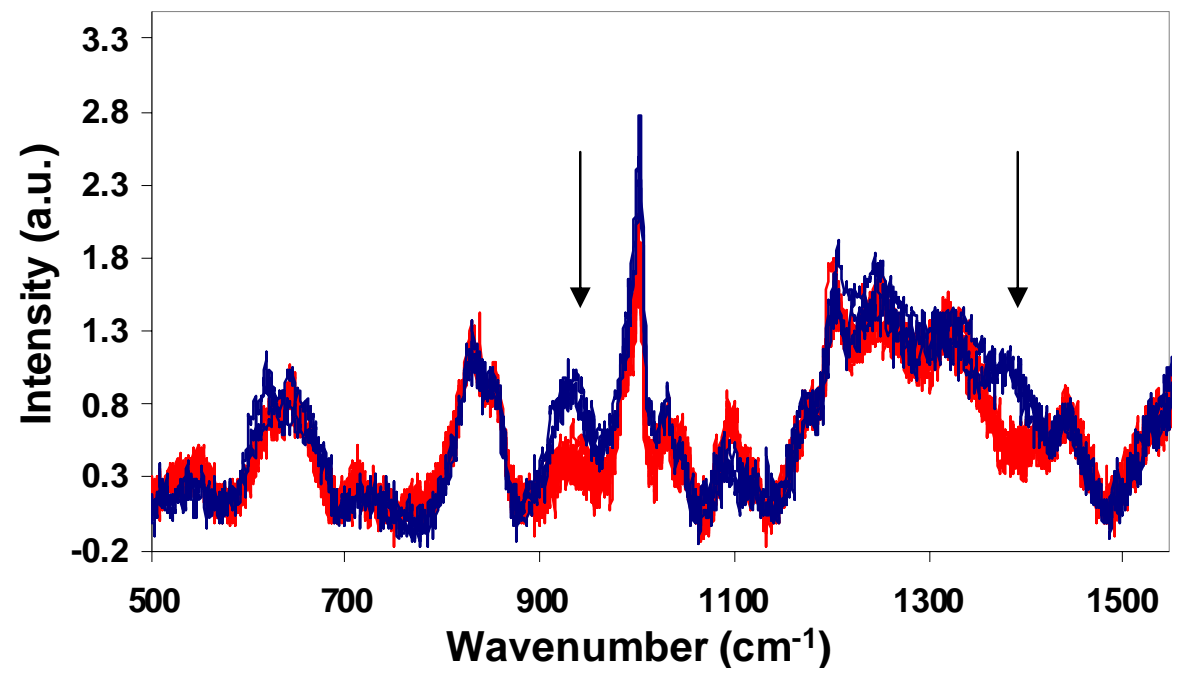

Fig. 7: Human insulin (blue) and insulin lispro (red) SERS spectra (collected over three spots each). Arrows indicate main spectra difference areas. 
Drachev et al, Figure 8
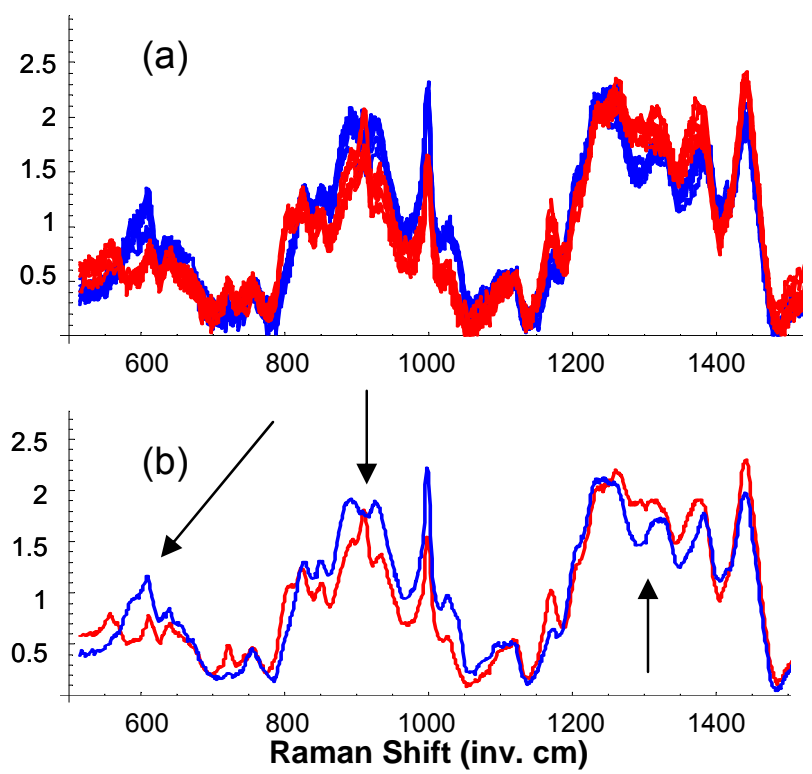

Fig. 8: SERS spectra of antigen (blue) and antigen after incubation in an antibody solution (red): a) Nine spectra, each obtained from three spots; b) Averages, with arrows indicating main spectra change areas 
Drachev et al, Figure 9

a) Ag/SiO2/glass

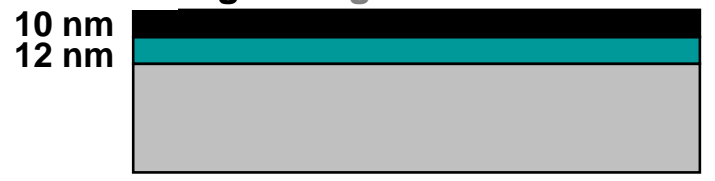

b) $\mathrm{Ag} / \mathrm{SiO} / \mathrm{Ag} / \mathrm{glass}$

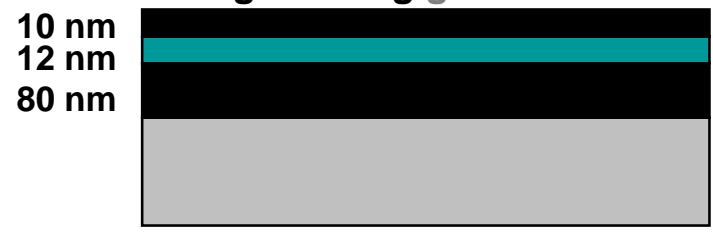

Fig. 9: Vertical layer structure for ASF substrates: (a) two-layer structure, (b) multi-layer structure 
Drachev et al, Figure 10

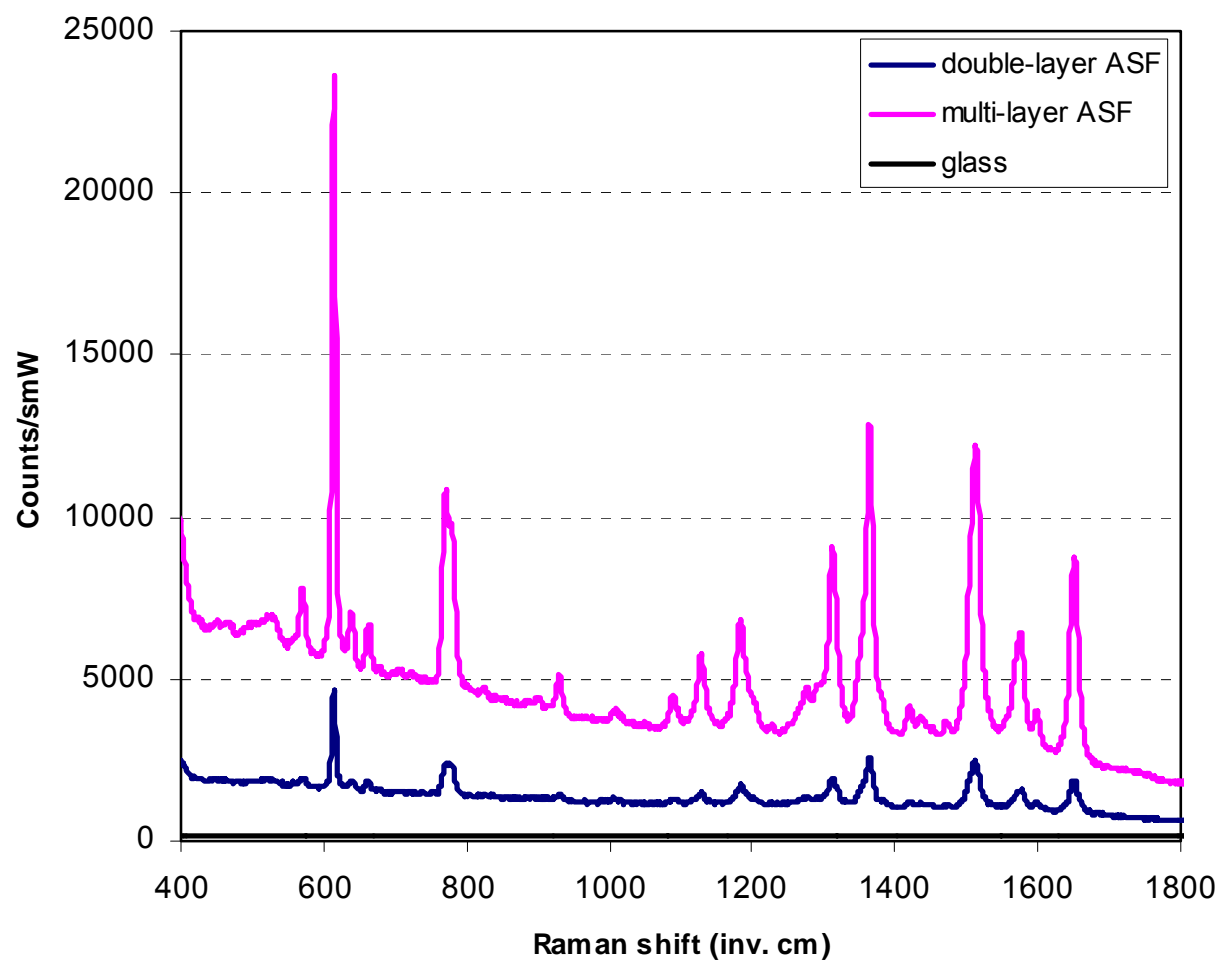

Fig. 10: R6G SERS spectra from double-layer and multi-layer ASF substrates 
Drachev et al, Table 1

Table 1: SERS signals for two types of ASF substrates (data is in counts per second per milliwatt)

\begin{tabular}{|l|c|c|}
\hline & $\begin{array}{c}\text { Two-layer } \\
\text { Ag/SiO } / \text { /glass }\end{array}$ & $\begin{array}{c}\text { Multi-layer } \\
\text { Ag/SiO } / \text { Ag/glass }\end{array}$ \\
\hline Insulin & Max $7 \mathrm{c} / \mathrm{smW}$ & $28-38 \mathrm{c} / \mathrm{smW}$ \\
\hline $\begin{array}{l}\text { Antibody } \\
\text { (anti-human interleukin 10) }\end{array}$ & $20-25 \mathrm{c} / \mathrm{smW}$ & $80-100 \mathrm{c} / \mathrm{smW}$ \\
\hline Antibody incubated with R6G & $3500 \mathrm{c} / \mathrm{smW}$ & $16000 \mathrm{c} / \mathrm{smW}$ \\
\hline
\end{tabular}

